\title{
Philosophiques
}

\section{Pour l'immobilisme?}

\section{Louise Poissant}

Volume 12, numéro 1, printemps 1985

URI : https://id.erudit.org/iderudit/203275ar

DOI : https://doi.org/10.7202/203275ar

Aller au sommaire du numéro

Éditeur(s)

Société de philosophie du Québec

ISSN

0316-2923 (imprimé)

1492-1391 (numérique)

Découvrir la revue

Citer cet article

Poissant, L. (1985). Pour l'immobilisme? Philosophiques, 12(1), 133-146.

https://doi.org/10.7202/203275ar

\section{Résumé de l'article}

Dans cet article, la question de l'immobilisme est abordée à la suite d'un détour par la description sommaire de diverses tendances féministes regroupées sous les catégories de féminismes de revendication, féminismes de libération et féminismes d'affirmation de la différence. Cette présentation permet de retracer une histoire des femmes et de leurs mouvements. À la suite de ce tour d'horizon, l'auteure essaie d'analyser le trait d'immobilisme dont on taxe les femmes et que l'on retrouve même, à des degrés et à des titres divers, dans de nombreuses thèses féministes. S'agit-il d'une caractéristique spécifiquement féminine ou cette constatation dépend-elle du lieu d'énonciation ? Quelques hypothèses sont envisagées pour ouvrir la question.
Ce document est protégé par la loi sur le droit d'auteur. L'utilisation des services d'Érudit (y compris la reproduction) est assujettie à sa politique d'utilisation que vous pouvez consulter en ligne.

https://apropos.erudit.org/fr/usagers/politique-dutilisation/ 


\title{
POUR L'IMMOBILISME ?
}

\author{
par Louise Poissant
}

RÉSUMÉ. Dans cet article, la question de l'immobilisme est abordée à la suite d'un détour par la description sommaire de diverses tendances féministes regroupées sous les catégories de féminismes de revendication, féminismes de libération et féminismes d'affirmation de la différence. Cette présentation permet de retracer une histoire des femmes et de leurs mouvements. À la suite de ce tour d'horizon, l'auteure essaie d'analyser le trait d'immobilisme dont on taxe les femmes et que l'on retrouve même, à des degrés et à des titres divers, dans de nombreuses thèses féministes. S'agit-il d'une caractéristique spécifiquement féminine ou cette constatation dépend-elle du lieu d'énonciation? Quelques hypothèses sont envisagées pour ouvrir la question.

ABSTRACT. In this article the issue of immobility is dealt with following a brief digressive description of the various femenists leanings, regrouped into categories of protest feminism, liberation feminism, and feminism assertive of difference. This presentation makes it possible to relate a history of women and their movements. Following this general survey, the author attemps to analyse the trait of immobility of wich women are accused and wich is found in many feminist argumentations to various degrees and under various labels. Is this a specifically feminine trait or does this observation depend on the context of uttering. Several hypotheses are considered in dealing with this issue.

On a dit de la femme qu'elle était immobile. Il s'agirait même d'après certains ( $\mathrm{E}$. Jones, M. Montrelay), d'un de ses traits spécifiques. Caractère relié à la concentricité de sa sexualité et de sa structuration psychique, trait de conservatisme associé à sa situation de dépendance (K. Millet), compulsion à la répétition engendrée par son origine même, son rapport archaïque à la mère (M. Montrelay, E.G. Belotti, C. Olivier), support de l'éternel féminin, plusieurs discours, féministes ou non, téaffirment par le biais d'instances qu'ils privilégient, qu'elles soient 
économique, psychanalytique, psychologique ou sociologique, cette thèse, " ce constat ", cet " état de fait " d'immobilisme. $\grave{A}$ l'heure des changements rapides, du virage technologique et des mutations qu'il entraine, au moment où les révolutions sont quotidiennes et vitales - «Plus je fais l'amour plus je fais la révolution " proclamait-on en mai 68 - alors que des peuples, des classes sociales, des penseurs, mettent tout en œuvre pour que ça change, les femmes, elles, anachroniquement, seraient immobiles. Pas exactement d'ailleurs puisqu'on le sait, elles ont embrayé le pas aux différents mouvements contestataires, elles ont entrepris, depuis le début du siècle, mais depuis une trentaine d'années en particulier des remises en question radicales. Paradoxe alors que cet immobilisme au cœur du changement ? Simple remue-ménage qui laisse en place l'essentiel ? Ou thèse qui renvoie à une écoute et à un point de vue immobiles ? Un bref aperçu des divers types de féminisme, de leur action et de l'élan qui les a portés permet de faire ressortir des éléments importants pour cerner la question.

L'histoire du féminisme regroupe différentes tendances qui dépendent des contextes dans lesquels elles émergent. On ne peut à proprement parler établir une succession des courants, bien que certains soient apparus après d'autres, puisque les situations des femmes sont très disparates et dépendent de leur distribution géographique et économique. Toutes les tendances sont donc en quelque sorte actuelles et leur présentation sommaire ne devrait pas laisser entendre que certaines orientations sont périmées. Sous toutes ses.formes, le féminisme a été et reste une atteinte à la catégorie qui sert de paradigme à l'humanité : le mâle, occidental, adulte, rationnel et blanc. Catégorie vide dans laquelle aucune variable ne trouve à s'inscrire, mais aussi et surtout, catégorie qui s'est élaborée tout au cours de l'histoire de l'Occident et qui s'est fixée depuis peu, à vrai dire depuis qu'elle est contestée. Par ailleurs, l'impulsion des interventions féministes n'a jamais été isolée. D'autres mouvements pour des motifs différents, mais pourrait-on dire, au nom d'une même vue et d'une même anticipation, et soulevés par une exaspération et une révolte aussi grandes, ont visé le modèle idéologique du mâle occidental ${ }^{1}$.

1. Il est intéressant de signaler que cette catégorie est très peu parlée en tant que modèle. Ce sont les groupes qui la contestent qui la nomment et la décrivent. Cependant, elle est 
En effet, déjà le féminisme des premières heures, celui qui exige une parité des droits avec les hommes, a mené une entreprise semblable à celle de plusieurs "minorités " opprimées. Les revendications des Noirs, celles des ouvriers, des minorités ethniques ou religieuses ont d'abord porté sur des questions d'équité. Plusieurs aspects de la démarche des femmes se retrouvent d'ailleurs dans les autres grandes causes. Ce féminisme, qui est surtout de revendication, réclame la reconnaissance de la femme sur les plates-formes publiques et des droits en tant qu'adulte et citoyenne à part entière. Un coup d'œil rapide sur la liste des droits demandés suffit pour rendre évident combien la femme était loin d'être considérée comme une personne à part entière. Pour ne parler que de ce siècle, en gardant en tête que les hommes avaient eux, ces droits, en voici quelques-uns : droit de vote ${ }^{2}$, le droit d'accès à la propriété, à l'éducation, droit au travail avec salaire égal pour des tâches égales, droit de disposer librement de son corps (avortement, contraception), droit à la parole, de fréquenter certains lieux publics, droit à son nom, à sa jouissance, etc. Bien sûr, dans cette perspective égalitaire, il s'agit de faire valoir que la femme, comme le Noir ou l'ouvrier, participent à l'humanité et que les différences sont inessentielles, qu'elles relèvent de l'histoire et de la culture. "On ne naît pas femme, on le devient " disait Simone de Beauvoir et une fois admis ce principe, les disparités de traitement apparaissent le produit d'une histoire réorientable. Ce qui est souhaité par ce type de féminisme, c'est l'appartenance à la catégorie valorisée, celle du mâle, occidental, adulte, blanc, rationnel, en mettant entre parenthèses l'épithète qui selon la voix revendicatrice, est accessoire : le qualificatif « mâle » pour les femmes par exemple. Les Nègres insisteront pour que "blanc" soit mis entre parenthèses, les jeunes contestaient l'épithète "adulte », etc.

présente en sous-entendu dans tous les énoncés sexistes et racistes et sa force idéologique est d'autant plus grande que sa circulation est voilée.

2. Le droit de vote n'a été obtenu au Québec qu'en 1940. Premier objet des revendications féministes organisées, d'abord en Angleterre par les suffragettes, il a exigé une telle énergie que les forces féministes sont tombées, en Angleterre et en France aussi bien qu'aux ÉtatsUnis lorsque le droit a été reconnu. Ce n'est que dans les années 50 que le féminisme se relèvera. Le deuxième sexe de $\mathrm{S}$. de Beauvoir date de 1949, La femme mystifiée de B. Friedman de 1963. 
Ce qui est en jeu c'est l'aspiration à une identité reconnue, au titre d'humain et pour la réussite de cette quête, il est essentiel de faire oublier les différences et de faire en sorte au contraire qu'elles soient atténuées, effacées, négligées, étant insuffisantes pour justifier la ségrégation ou les inégalités qu'elles engendrent.

La deuxième forme de féminisme, celui de la libération, s'apparente à la forme précédente mais insiste danvatage, sous l'influence des sciences sociales, sur l'aliénation et l'oppression qu'ont connues les femmes au cours des siècles. Lié aux grandes causes de libération, ce féminisme a adopté un vocabulaire qui imprime à cette démarche un caractère politique universel. $M$. Yaguello recense quelques-unes des expressions fréquemment utilisées et qu'on retrouve dans la plupart des mouvements de libération ${ }^{3}$ : exploitation (de la femme par l'homme), groupe dominant/groupe dominé, lutte contre l'oppresseur, femmes en lutte, lutte des femmes, la cause des femmes (sur la cause des peuples), condition féminine (conditions des Noirs, condition ouvrière), fémellitude, féménitude, maternitude (sur négritude). On pourrait ajouter que le nom même de plusieurs regroupements est calqué de façon explicite sur des mouvements politiques : M.L.F. par exemple ou Women's Lib et F.L.F. ${ }^{4}$ Certains titres d'ouvrages sont aussi des références claires : Le féminisme ou la mort (sur le slogan de la révolution française " la liberté ou la mort "), Féminisme et Révolution ${ }^{5}$. Volontiers solidaire d'autres groupes, ce féminisme cherche à réaliser une libération globale et bien qu'il soit depuis quelque 20 ans indépendant des partis socialistes ou communistes, la lutte qu'il mène vise le même ennemi, ponctuellement le capitalisme et derrière lui l'oppresseur en général. C'est dans cet esprit que S. Rowbothan ${ }^{6}$ milite pour que soient reliées les différentes forces révolutionnaires et pour qu'elles neutralisent et déjouent l'identité que le capitalisme renvoie des groupes révolutionnaires. Dans ce contexte, la lutte des femmes est un cas particulier de la révolution générale. K. Millet ${ }^{7}$ radicalisera cette position en expri-

3. Yaguello, M. Les mots et les femmes, Paris, Payot, 1978.

4. M.L.F. = Mouvement de libération des femmes. F.L.F. = Front de libération des femmes.

5. D'Eaubonne, F., Le féminisme ou la mort, Paris, P. Horay, 1974 et Rowbothan, S. Féminisme et Révolution, Paris, Payot, 1973.

6. Rowbothan, S. Conscience des femmes, monde de l'bomme, Paris, ed. des Femmes, 1976.

7. Millet, K. La politique du mâle, Paris, Stock, 1971. 
mant la tension et les antagonismes entre les genres mâle et femelle en termes de politique sexuelle. Les sexes sont non seulement inscrits dans des déterminismes socio-culturels comme d'autres ${ }^{8}$ l'avaient montré, mais de plus, leurs rapports sont affaire de pouvoir, ils sont de nature politique. Mouvement de libération ou de révolution sexuelle comme dira encore $\mathrm{K}$. Millet. À ce régistre, la femme a embrayé le pas à l'ère des révolutions que l'on retrouve un peu partout, sur la scène politique, sociale aussi bien qu'en économie. On parle de révolution technologique, de révolution sexuelle, de révolution tranquille au Québec, des révolutions épistémologiques, informatiques. On organise même des publicités de produits ménagers en les qualifiant de révolutionnaires ou nouveaux. Il n'est pas inopportun de signaler ici que ces publicités s'adressent aux femmes au foyer et que les images qu'elles véhiculent renforcent par ailleurs des attitudes traditionnelles, un des visages de l'immobilisme.

Il faut cependant rappeler que le féminisme de libération, pour des raisons de stratégie et d'éveil de la conscience féministe, a associé la femme à l'ensemble des minorités opprimées et a maintenu, c'était le prix à payer, la femme et l'homme, dans la dialectique du pouvoir, dans des rapports d'oppression, dans la position de victime et de tortionnaire. Prise de conscience douloureuse et historiquement nécessaire mais en même temps idéologiquement limitée aux termes mêmes de la relation de pouvoir. Cette entreprise comporte le risque de développer et de renforcer des comportements de victime, bien plus que de réaliser, ce que certains ont craint, fantasme aussi peu réaliste que provocateur, que les femmes renversent le pouvoir et en deviennent les gérantes. Mais une méfiance et parfois mêrne une haine à l'égard du mâle sont apparues et sont même encouragées? dans certains milieux, sentiments qui vont souvent au delà de ce qu'il y avait à contester et qui durent. Bien sûr, il est essentiel de lire historiquement les rapports hommes-femmes en termes de pouvoir. C'est d'autant plus important que cette lecture per-

8. La grande majorité des féministes a adopté un point de vue historiciste qu'elle applique à l'une ou l'autre des dimensions de la vie des femmes.

9. Certaines féministes, des radicales ont exprimé ce désir mais elles ne sont pas représentatives de l'ensemble des mouvements. 
met de rejoindre les femmes qui sont, par leur rôle dans la famille, isolées de la scène publique et dispersées, ce qui représente la condition la plus propice à la manipulation. Mais il serait coûteux d'hypostasier cette lecture et d'en faire l'unique instrument de la remise en question des rôles sexuels. L'approche politique risque en effet de maintenir et de fixer les relations hommes/femmes sous un mode antagoniste. Pour éviter l'insularisme et l'isolement des femmes, d'autres approches sont de plus en plus valorisées dont les arts et la littérature qui permettent d'atteindre un grand nombre de femmes.

Ce qui caractérise donc ce type de féminisme, à l'instar d'autres regroupements, les groupes étudiants et contestataires en général, c'est la charge globale qu'il porte contre le modèle du mâle, blanc, occidental . . . Il ne s'agit plus simplement de mettre entre parenthèses l'une ou l'autre des caractéristiques pour arriver ainsi à s'identifier au modèle dans l'ensemble, mais plutôt de chercher à miner le modèle, précisément parce qu'il apparaît étouffant et surtout, instrument de ségrégation et d'oppression. Ce qui éclate avec et derrière cette catégorie, ce sont les valeurs d'un système qui ne correspond plus aux aspirations de plusieurs groupes sociaux.

Le troisième type de féminisme est en quelque sorte un des produits du féminisme de libération. On pourrait l'appeler féminisme des différences : par des théories les femmes nomment leur spécificité, affirment leur identité dans ce qu'elle a d'autre. Les psychanalystes L. Irigaray et $\mathbf{M}$. Montrelay entre autres se sont consacrées à ce dévoilement. Critiquant et rejetant la définition de la femme que Freud dessine au travers son traitement de la sexualité féminine, sexualité de castrée mue par l'envie du pénis, Irigaray démontre comment "un vieux rêve de symétrie " ${ }^{10}$ entre homme et femme équivaut à la négation de la spécificité de la femme. Cette dernière n'apparaît que le négatif de l'homme, qui sert, sur cette scène encore, de paradigme à l'humanité. Pour Iragaray, la femme se caractérise par un corps sans organe ${ }^{11}$. La syntaxe du féminin ne serait pas de l'ordre des oppositions sujet-objet, pas plus qu'elle ne se rattacherait au

10. Titre d'un chapitre de Speculum, Paris, Minuit, 1974.

11. Irigary, L. Ce sexe qui n'en est pas un, Paris, Minuit, 1977, p. 138. 
sens et au nom propre. Puisque la féminité n'est pas une, plus d'appropriation mais plutôt une proximité. Le proche relaie du propre. En effet, la femme est deux sexes (clitoris et vagin) ${ }^{12}$ et son anatomie intime rend continuellement possible un autoérotisme et une jouissance sans instrument, sans objet. Plus proche d'une mécanique des fluides que des solides puisque ça coule d'elle, la femme est toujours en défaut ou en excès par rapport à l'un, à l'unité ${ }^{13}$. Pas une mais double et plurielle la femme est jouissance, "black out du sens $"{ }^{14}$. Ce qui distingue la femme, ce qui commande chez elle un rapport au monde tout autre, c'est cette différence sexuelle abyssalle entre elle et le mâle.

M. Montrelay, conformément à la position psychanalytique place aussi le lieu essentiel ${ }^{15}$ de la différence entre hommes et femmes au niveau du sexuel. Pour elle, la spécificité de l'inconscient féminin dépend de la coexistence incompatible entre le phallocentrisme inspiré de Freud et le concentrisme. Le concentrisme correspond à la thèse de l'École anglaise, celle de Jones en particulier et rend compte de la sexualité féminine à partir de la configuration bouche - anus - vagin : orifices qui visent l'incorporation et l'introjection, qui représentent le « faire sien " l'acte de dévorer ${ }^{16}$ et qui rappellent et réactivent le plaisir infantile de la succion. Montrelay insiste par ailleurs sur la difficulté épistémologique aussi bien qu'objective de définir le féminin. Parler de continent noir à propos de la sexualité féminine ne serait pas qu'un aveu d'ignorance. Au delà, il s'agirait d'un trait caractéristique de la femme. En effet, la sexualité de cette dernière serait irreprésentable, exclue de l'ordre symbolique parce que non refoulée, inapte au refoulement. Ayant eu une sexualité moins réprimée dans l'enfance et réactualisant par sa jouissance le plaisir infantile et le rapport au corps de la mère, la femme ne vivrait aucune menace de castration, n'aurait pas à faire le deuil des pulsions infantiles, n'aurait donc rien à

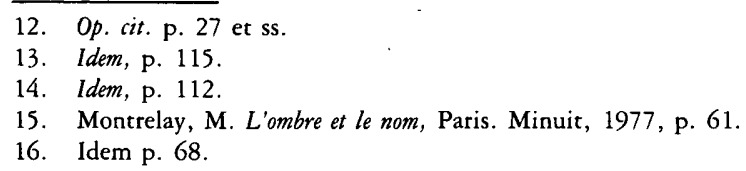


refouler ${ }^{17}$. Cherchant à opérer la jonction entre Freud et Jones, Montrelay conclura qu'alors que la parole de l'homme tout comme sa sexualité sont à l'œuvre dans un horizon de séparation, de projection hors de soi, dans un horizon de mort, dont la détumescence pénienne serait la métaphore, la femme, elle, aurait un corps propre, corps de jouissance dans l'immédiateté, corps qui vit, dans l'ombre de la jouissance de la mère, le nom du père. Maintien du phallocentrisme cependant : la femme continue de désirer le pénis, non pour elle-même mais pour l'autre, pour assurer la valeur du phallus paternel, pour garantir le désir parental. La part de phallocentrisme de la sexualité de la femme ne serait donc pas un négatif de l'homme, mais deviendrait plutôt ce qui constitue la valeur même du phallus.

Ces deux théories psychanalytiques ont été développées un peu plus longuement parce que leur position est peut-être un peu moins connue que celles des autres féministes convoquées ici et parce qu'elles présentent de façon exemplaire le féminisme des différences. En effet, les thèses de la différence, féministes ou non, essaient de faire valoir le trait spécifique, celui qu'on mettait entre parenthèses, en l'articulant par des approches complexes, psychanalytique, anatomique, socio-politique, etc. Il est important de signaler que très peu de discours valorisant la spécificité exploitent un aspect unique de différenciation. Par ailleurs, le biologique qui a souvent servi d'argument de ségrégation et qui était évacué et occulté par les féministes des deux tendances précédentes, est réintroduit et conjugué avec les autres instances constitutives. Mais ce qu'il faut surtout remarquer c'est que la plupart des thèses de la différence suggèrent que le trait distinctif est exportable. À l'heure du "Black is beautiful", on renoue avec ses origines ethniques. La rationalité est encore prégnante mais on nous apprend que chacun est un peu fou et qu'un enfant vit dans chaque adulte. Nous sommes tous bisexuels et

17. Pour Montrelay, la sexualité de la femme serait irreprésentable, exclue de l'ordre symbolique parce que non refoulée, inapte au refoulement. Parce qu'elle aurait moins connu d'interdits face à la masturbation infantile, ses organes étant moins apparents, par ailleurs, à cause précisément de la concentricité de sa sexualité qui inscrit sa jouissance dans le sillage des pulsions archaïques et enfin parce que son corps est rappel du corps de la mère, corps érotique puisque corps d'une autre femmme qui est aimée au travers le corps propre, corps phallus alors, pour ces trois raisons, la femme n'aurait rien à perdre, ne connaîtrait aucune menace de castration. 
donc nous ne sommes pas forcément destinés à reproduire des petits mâles, adultes, occidentaux, blancs. En rendant exportable le trait spécifique, les porte-parole des minorités ethniques, les professionnels de la santé mentale, les homosexuels et les psychologues de l'enfance ont non seulement sorti des minorités de leur ghetto, mais ils ont de plus miné le modèle du mâle . . .

Les thèses féministes commencent elles aussi à exporter ce qu'elles ont découvert de spécifique chez la femme. Montrelay dira que le corps propre, le corps de jouissance, le corps en creux, maternant, figure dans le désir de l'homme. À l'occasion de la présentation d'un cas ${ }^{18}$ elle parle du "féminin du père ". Irigaray avance que le corps sans organe, corps à la jouissance plurielle, peut être vécu, ne serait-ce que fantasmatiquement ${ }^{19}$ par les hommes. Mais au-delà de l'intérêt que présente l'exportabilité du trait spécifique en tant qu'il érode le modèle traditionnel, il est essentiel pour maintenir ouverts et possibles les liens entre les diverses différences. En effet, les différences maintenant affirmées par les " minorités ${ }^{20}$ engendreraient une violence aussi grande que celle qu'elles combattent, non pas tant parce qu'elles finiraient par reproduire du même, cette menace est bien peu réaliste, mais parce qu'elles reproduiraient des rejets. L'affirmation de la différence peut discréditer le vis-à-vis, peut servir d'argument pour interrompre l'échange. Déclarer qu'étant donné sa différence propre l'autre ne peut pas comprendre, agit aussi violemment que l'énoncé qui rejetait au nom de la différence de l'autre. Ce type d'intervention rend impossible pour l'interlocuteur d'enchaîner et accule au silence ${ }^{21}$. L'exportabilité, la possibilité de reconnaître chez l'autre du même et non pas le même, bien entendu, et éventuellement pourrait-on souhaiter,

18. Op. cit. p. 142 et le cas Louise.

19. Irigaray, Ce sexe . . p. 138.

20. On peut se demander qui n'est pas minoritaire aujourd'hui, au moins par certaines fonctions et par certains traits. En tout cas nous le sommes tous à certains âges.

21. Le féminisme de libération a rendu manifeste la difficulté de dialoguer entre hommes et femmes. Il a répondu à la violence séculière que subissaient les femmes dans l'impossibilité de parler où elles étaient cantonnées. La méfiance à l'égard de l'oppresseur a discrédité la parole de ce dernier et l'a mis en position de ne plus pouvoir enchaîner sur la parole de l'autre. Le féminisme des différences pourrait répéter le geste violent qui accule l'autre au silence. Étant donné la différence, l'autre ne pourrait être partenaire du dialogue puisqu'il ne comprend pas. Les femmes ont bien connu cette situation : on ne les consultait pas parce qu'elles ne pensaient pas, elles n'entendaient rien aux affaires publiques, au monde de l'homme. 
la possibilité de découvrir en soi du même de l'autre, convie à la coexistence dans le respect et l'ouverture des multiples différences. Il ne suffit pas, bien que ce soit toutefois essentiel, de faire valoir sa différence, il faut aussi se rendre disponible à celle des autres. Le narcissisme des minorités ne doit pas insulariser, même par choix, parce qu'alors il répéterait ce qu'il devait combattre, l'isolement auquel le modèle uniformisant cantonne lorsqu'on n'est pas dans les rangs. Dans une telle perspective, "l'empire du même » que contestait Irigaray ferait plutôt figure de château de cartes, fragile et inhabitable ${ }^{22}$.

Mais que nous révèle ce trop long et trop sommaire détour historique ? Comment peut-on dire de la femme qu'elle est atteinte d'immobilisme alors que manifestement, elle a été liée à tous les changements qu'a connus le $\mathrm{XX}^{\mathrm{e}}$ siècle, alors qu'elle a accepté l'insécurisante mais exaltante démarche qui consiste à remettre son identité en question ? Il apparaît que les différentes thèses de l'immobilisme ne fonctionnent pas parce qu'elles isolent un aspect de l'activité ou de la texture de la femme, aspect qui se trouve figé alors. Un rapide coup d'œil sur ces diverses thèses est instructif. Montrelay parle d'immobilisme de la femme notamment à propos de l'envie du pénis. On a vu comment cette envie était un effet de la valorisation que la fillette fait du phallus paternel, précisément parce que le pouvoir qui y était associé s'est trouvé mis en cause. Pour renforcer le prestige paternel menacé, la fille adopterait des attitudes passives, d'attente, d'impuissance afin de signifier que sans pénis on n'est rien, mais surtout que sa possession est argument de prestige. Par ailleurs, la concentricité de la sexualité féminine, parce qu'elle relève d'orifices captateurs et parce qu'elle réactive la jouissance infantile, réduirait la femme à la répétition et à la reproduction, face à la parole et avec ses objets, ses enfants par exemple, à une même attitude avide et captatrice, enveloppante et dévorante. Cette constatation est aussi relevée par d'autres psychanalystes reconnues féministes. C. Olivier ${ }^{23}$ entre autres confirme la position de Montrelay en ce qui concerne la parole. D'après cette

22. Il faut signaler que le modèle a aussi été critiqué du lieu de la philosophie. Les entreprises de Deleuze, Derrida et Lyotard entre autres, en sont de magnifiques déconstructions. Nietzsche avait déjà donné le ton.

23. Olivier, C. Les enfants de Jocaste, Paris, Denoël-Gonthier, 1980. 
psychanalyste, la femme répète compulsivement une demande d'amour et d'attention originairement adressée à la mère et frustrée et ultérieurement dirigée sur l'homme mais à jamais insatisfaite, quelle que soit la réponse masculine. Il faut peut-être signaler que pour Olivier l'homme est acculé à répéter de son côté l'attitude de fermeture qu'il a adoptée lors du désinvestissement libidinal du corps de la mère. Ces deux thèses, pour justes qu'elles soient n'en demeurent pas moins partielles à ce sujet. Les conceptions de la configuration maternelle et en particulier de l'instinct, cette mamelle de conservatisme féminin, ont été secouées par les considérations socio-économiques que E. Badinter ${ }^{24}$ a apportées.

En effet, est-ce par hasard qu'apparaissent en même temps en France, la première école agronome, la médecine vétérinaire et la première école de sages-femmes ? Selon le contexte, le rapport à l'enfant semble plus ou moins valorisé, encouragé. Et l'instinct maternel apparaît bien plus comme étant un effet des valeurs agraires et traditionnelles entre autres, qu'une instance naturelle et universelle. Bien sûr, le niveau d'intervention de Badinter n'est pas le même et le conservatisme qu'elle interroge n'est pas de même nature que l'immobilisme dont parle Montrelay. Mais précisément, ce qu'on pourrait souhaiter c'est que les voies explicatives se multiplient devant les phénomènes pour ne pas figer les complexes considérés sous une seule vue.

De même, le conformisme et l'esprit traditionnel dont on taxe les femmes, attitudes que l'on retrouve en particulier dans le rapport à la langue - les femmes étant les dépositaires de la langue maternelle qu'elles parlent et écrivent plus correctement que les hommes (Yaguello) - et dans le rapport à la chose publique, politique - les femmes ayant majoritairement un vote et des opinions moins progressistes - mériteraient une analyse plus serrée que celle qu'on en fait habituellement. $\mathrm{K}$. Millet $^{25}$ attribue ce conservatisme au sentiment de dépendance que la femme éprouve à l'égard de son pourvoyeur. Sa survie et sa sécurité lui apparaissent liées à la prospérité de ceux qui la nourrissent. Cette vassalité a tendance à persister d'ailleurs, même

24. Badinter, E. L'amour en plus, Paris, Flammarion, 1980.

25. Millet, K. op. cit. p. 53. 
lorsque la femme travaille et est économiquement autonome. L'habitude d'occuper une position sur la plate-forme publique changera sans doute cette manière d'être et il est inutile d'insister sur la contribution du féminisme pour changer cet état de fait. On retrouve aujourd'hui d'ailleurs un grand nombre de femmes dans les partis politiques les plus à l'avant-garde, les partis écologistes par exemple. Cependant, ce qui est omis lorsqu'on analyse le conformisme politique des femmes, c'est que si elles ont tendance à répéter des comportements conservateurs, le fait même de les répéter est encore plus fondamental et significatif et à cet égard, l'homme, son vis-à-vis, répète aussi. Le mouvement est nécessairement dialectique et implique conjointement chacun des termes.

Par rapport à la langue, le phénomène, on s'en doute, est encore plus complexe. Bien sûr, cette langue maternelle est celle de l'Autre, celle du père et dans l'attitude conservatrice de la femme, ce facteur est sans doute déterminant. Plusieurs paramètres socio-économiques et culturels - le fait que les femmes composent la grande majorité du public lecteur, le fait qu'on ait orienté pendant longtemps les filles dans des études littéraires plutôt que dans des domaines scientifiques, le fait qu'on ne stimulait pas les filles à être inventives ou créatrices, dans les minorités linguistiques, le fait que les femmes soient moins en contact avec l'extérieur et la langue dominante, etc, - rendent compte au moins en partie du phénomène. Une analyse du conservatisme face à la langue, analyse qui ne peut être que suggérée ici, doit aussi étudier le rapport souvent répétitif à la parole. M. Duras et X. Gauthier constatent cette dimension de leur échange dans Les Parleuses ${ }^{26}$. Duras est même tentée d'en faire un trait de la spécificité féminine. Olivier et Montrelay n'hésitent pas à relier la parole qui répète des demandes et l'attitude d'appropriation du langage à une configuration psychique particulière aux femmes. Bien sûr, parallèlement à ces constats, il y a tout le travail d'écriture et d'innovations qu'ont réalisé les femmes depuis une vingtaine d'années. Cette question est même au cour d'une bonne part de la production actuelle, théorique

26. Duras, M. et Gauthier, X. Les Parleuses, Paris, Minuit, 1974. 
ou littéraire des femmes, production qui est renouvelée et éloignée du modèle traditionnel.

Mais comment et pourquoi toutes ces thèses sur l'immobilisme ? Thèses, faut-il le signaler, qui passent le plus souvent inaperçues, qui sont présentes et actives sans être très remarquables. Si les femmes font partie d'un des groupes sociaux qui a le plus bougé et évolué depuis une trentaine d'années, si par ailleurs, plusieurs de leurs comportements répétitifs sont dialectiquement liés aux rôles tout aussi répétitifs des hommes, comment expliquer qu'on insiste sur l'immobilisme féminin ? Diverses raisons sont à envisager. En voici quelques-unes à titre d'hypothèses. D'abord il semble qu'on relance cette problématique au moment où on peut observer de profonds changements chez les femmes et les groupes de femmes, comme s'il fallait, quelque part, que la femme ne change pas. Dépositaire de l'espèce humaine, sa crise d'identité menacerait peut-être, inconsciemment bien sûr, le rapport à la génération, à la reproduction et plus globalement à la vie même. Instance biologique refoulée par les féministes elles-mêmes, elle resurgirait au moment où économiquement et socialement les femmes se libèrent, au moment où l'on accepte que leur aliénation soit affaire de culture et non de nature. Par ailleurs, pour l'humain moderne qui a subi et provoqué un grand nombre de changements et qui s'éprouve à plusieurs égards, déraciné et déconnecté, isolé et désorienté, la perspective de l'immobilisme de la femme, trait qui lui serait spécifique et à la limite " naturel », représenterait un havre rassurant, voire nécessaire. La femme repos du guerrier adaptée aux nouveaux agresseurs que sont le stress, la névrose, l'insécurité sociale. La nature même de certains changements y est aussi sans doute pour quelque chose. En effet, dans la mesure où les modifications ne s'opèrent plus par le biais de revendications calquées sur le modèle mâle mais par des déplacements en profondeur qui portent sur la prise en charge de soi, le rapport aux institutions, à l'environnement, à la communication en général ; dans la mesure où certaines attitudes ne font pas que déplacer des termes d'une dialectique mais introduisent de tout nouveaux rapports, les points de repères manquent pour mesurer ou juger la nouvelle situation. Ce qui échappe ou dépasse, le différent est souvent réduit à l'oubli ou au silence. Et puis, on 
a peut-être besoin de désigner la femme comme immobile parce que le changement ne se mesure que dans le sentiment qu'on a de changer. Nommer la femme immobile serait alors la seule façon pour l'homme ou pour l'autre, de se percevoir changeant. Ainsi, une façon de voir ou d'entendre, toujours la même, figerait la parole de la femme et figerait sa réalité, l'immobiliserait.

Bien sûr, on pourrait envisager selon une autre perspective le trait d'immobilisme dont on taxe la femme. Cette attitude ne se limiterait pas au conservatisme qu'on lui associe généralement. Le goût du présent, l'assomption de ce qui est, aspects qui la caractérisent aussi et qui par ailleurs se conjuguent bien avec une éthique actuellement très populaire, ne sont pas du tout retenus par les thèses présentées ici. On parle plus volontiers de statisme que de stabilité, de conservatisme que de contentement, de stagnation que d'adaptation. On pourra objecter que les versions connotées positivement ne correspondent pas à la situation des femmes ou à l'image qu'elles en donnent. Il est néanmoins important de signaler que l'étiquette d'immobilisme conserve une coloration morale et péjorative. Par ailleurs, ce qui s'oppose à l'immobilisme, n'est pas également considéré et estimé. La mobilité, l'action et l'évolution auxquelles on pense le plus facilement, ne sont pas les seules figures. L'agitation, le changement pour le changement, changement qui n'est symptôme finalement que du fait que rien n'a véritablement changé, sont aussi des formes qu'a adoptées la volonté de sortir du traditionnalisme et du conformisme. Et les femmes, dans leurs mouvements, n'ont pas échappé à ces pièges. Mais au bilan global, tout porte à croire que dans l'idéologie actuelle, là où il semble intéressant d'être immobile, la femme n'y est pas, alors qu'on l'y inscrit, à l'aide de l'appareil théorique et du féminisme même, là où c'est connoté péjorativement. Ce qui m'incite à suggérer que plus ça change, plus ça veut changer...

Département de philosophie

Collège de St-Laurent 